\title{
Postoperative complications (early and late) after pediatric cataract surgery in Sohag University Hospital
}

\author{
Hany F. Mohammed, Ismail M. Abd El-Latif, Ali M. Ismail and Hatem G. Ammar \\ Department of Opthalmology, Sohag Faculty of Medicine, Sohag University
}

\begin{abstract}
Introduction: Glaucoma is a recognized complication of pediatric cataract surgery. Despite improved surgical techniques, the incidence of glaucoma following successful cataract removal remains high. A significant number of surgeons regard aphakia as a cause of glaucoma. This glaucoma, however, may be better described as 'glaucoma in aphakia and pseudophakia'. Other complications, retinal detachment. It was found that retinal detachment is infrequent following aphakia in pediatric cataract surgery, at least at short term follow-up. Corneal astigmatism is recognized as a problem arising from cataract surgery.

Aim of the work: To evaluate postoperative complications (early and late) after pediatric cataract surgery in Sohag University Hospital

Patients and Methods: A prospective, randomized study, patients were divided into 3 groups based on the surgical technique they had undergone. Group A "25 patients" : patients who have undergone Lensectomy anterior vitrectomy (LAV). GroupB"24patients": patients who have undergone Extracapsular cataract extraction, primary posterior capsulorhexis or capsulotomy, anterior vitrectomy, and IOL implantation (ECCE/PPC/AV/IOL). Group C"26patients": patients who have undergone Extracapsular Cataract Extraction and IOL Implantation (ECCE/IOL)

Results: Early postoperative complications were diagnosed in 34 eyes (35.1\%). Postoperative iritis occurred in 7 eyes $(7.2 \%)$ with mild inflammatory reaction. Striate keratopathy was found in 4 eyes $(4.12 \%)$ and cleared after frequent topical steroid application. Postoperative endophthalmitis: occurred in one eye in group A in the second postoperative day. Twenty-four hours later, 3 ports pars plana vitrectomy was done but unfortunately the eye passed into atrophia. Early IOL capture occurred in 2 eyes $(2.1 \%)$ with PMMA IOL. Both patients belonged to group C. Both occurred in the second postoperative day. Postoperative retinal detachment (RD) occurred in one eye $(1 \%)$ in group B, Posterior capsule opacification (PCO) occurred in 30 eyes (31\%) during the first 3 months of follow up. Seven eyes (7.2\%) belonged to group A. In group B, 5 eyes $(5.1 \%)$ had PCO, Late IOL capture occurred in 9 eyes $(9.3 \%)$. These included 5 eyes $(5.2 \%)$ in group B; among them 4 eyes $(4.1 \%)$ had been implanted PMMA IOL.
\end{abstract}

Conclusion: Complications after cataract surgery in children are often associated with a robust inflammatory reaction or secondary opacity and, in infants, glaucoma.

Key words: Cataract, Complications, Sohag university.

\section{Introduction}

Pediatric cataract is the most common cause of treatable childhood blindness, accounting for $5-20 \%$ of blindness in children worldwide. Managing cataracts in children remains a challenge. Treatment is often difficult and tedious and requires a dedicated team effort, the most important members being parents ${ }^{(\mathbf{1})}$.

The following complications occur frequently during pediatric cataract surgery. Visual axis opacification 
VAO still remains the most frequent complication of pediatric cataract surgery ${ }^{(\mathbf{1})}$. The most critical factor influencing the occurrence of VAO is age. While opacification is nearly universal in infantile eyes, the incidence decreases with increasing age. Primary management of the posterior capsule and anterior vitrectomy are effective in preventing reopacification of visual pathways. The type and material of the IOL is another factor affecting the incidence of VAO. A new IOL implanted using an innovative 'bag-in-the-lens' technique seems promising in greatly reducing or eliminating PCO. A Sealed Capsule Irrigation device has a potential clinical usefulness in reducing the incidence of VAO, which needs to be confirmed in pediatric eyes ${ }^{(2)}$.

Glaucoma is a recognized complication of pediatric cataract surgery. Despite improved surgical techniques, the incidence of glaucoma followingsuccessful cataract removal remains high. A significant number of surgeons regard aphakia as a cause of glaucoma. This glaucoma, however, may be better described as 'glaucoma in aphakia and pseudophakia'. The most common type of glaucoma to develop following congenital cataract surgery is open-angle glaucoma. The risk factors include age at surgery, preexisting ocular abnormalities, type of cataract and the effect of lens particles, lens proteins, inflammatory cells and retained lens material. In addition, microcornea, secondary surgery, chronic postoperative inflammation, the type of lensectomy procedure or instrumentation, papillary block and the duration of postoperative observation have been found to influence the likelihood of glaucoma after pediatric cataract surgery. Undergoing lensectomy at a young age, especially in the first year of life, may be a risk factor for development of glaucoma. It has been suggested that the immaturity of the developing infant's angle leads to increased susceptibility to secondary surgical trauma. Hence some surgeons believe it prudent to consider delaying surgery until the infant is 4 weeks old in bilateral cases. Glaucoma can occur at any time after congenital cataract surgery. Therefore, pediatric aphakic and pseudophakic patients should be routinely monitored for glaucoma throughout their lives ${ }^{(2)}$.

Uveal inflammation Intense uveal inflammation or severe fibrinoid reaction is a concern particularly in infants and younger children. Addition of heparin to the irrigating solution has been suggested to reduce postoperative inflammatory reaction and related complications such as synechiae, pupil irregularity and IOL decentration ${ }^{(3)}$.

Other complications, retinal detachment. It was found that retinal detachment is infrequent following aphakia in pediatric cataract surgery, at least at short term follow-up. Corneal astigmatism is recognized as a problem arising from cataract surgery. Postoperative astigmatism is of greater importance in children than in adults because of its adverse effect on vision development and the risk of amblyopia. Children, however, show spontaneous regression of astigmatism during the first 5 months of follow-up (4)

An interesting finding is that relatively less astigmatism was observed in children having surgery under 3 years. Eye growth and changing refraction Predicting axial growth, and the refractive change that accompanies it, is one of the major challenges for long term care following pediatric cataract surgery. This is especially true with wide-spread acceptance of fixed power IOL implantation. Unless the growth of the 
eye can be accurately predicted, selection of IOL power is a difficult task. Axial growth after cataract surgery can be attributed to normal eye growth, other factors - including age at surgery, visual input, the presence or absence of IOL, laterality, genetic factors - also influence this process ${ }^{(4)}$. The interocular axial length difference was found to be another important variable influencing axial growth. Understanding pediatric eye growth will help in IOL power calculation and the prediction of refractive changes after IOL implantation ${ }^{(4)}$.

\section{Aim of the work:}

This study aimed to evaluate postoperative complications (early and late) after pediatric cataract surgery in Sohag University Hospital

\section{Patients and Methods:}

\section{Design:}

A prospective, randomized clinical study.

\section{Patients:}

Out of 116 patients (142 eyes) who started the study, only 75 patients (97 eyes) completed the 2-years follow up period and their data were analyzed and presented here.

This study was conducted in the Ophthalmology Department, Sohag University Hospital in the period from February 2008 to June 2013.

\section{Methods:}

Patients were divided into 3 groups based on the surgical technique they had undergone.

- Group A "25 patients" : patients who have undergone Lensectomy anterior vitrectomy (LAV).

- Group B"24patients": patients who have undergone Extracapsular cataract extraction, primary posterior capsulorhexis or capsulotomy, anterior vitrectomy, and IOL implantation (ECCE/PPC/AV/IOL).
- Group C "26patients": patients who have undergone Extracapsular Cataract Extraction and IOL Implantation (ECCE/IOL)

\section{Preoperative evaluation}

- Complete medical history and physical examination

- Complete ophthalmological evaluation, including:

a. Visual acuity assessment (if possible)

b. Anterior segment examination by slitlamp

c. Fundus examination after pupillary dilatation (if cataract density allowed) to exclude associated posterior segment anomalies

d. IOP under general anesthesia.

e. Ultrasound examination: (A- and Bscan) was performed under sedation or UGA to exclude posterior segment pathology

\section{Inclusion criteria:}

- Patients aged from birth to 12 years.

- Patients with pediatric cataract; including congenital, developmental and post-traumatic cataract after blunt trauma.

- Patients with a follow up period of at least 2 years after surgery.

- Patients fulfilling the previous criteria and followed the protocol of management of this study.

\section{Exclusion criteria:}

- Patients with traumatic cataract after penetrating trauma were excluded from the study.

- Patients with a follow up period of less than 2 years after surgery were excluded from the study.

- Patients with associated posterior segment anomalies on fundus examination. 


\section{Results}

The mean age of our patients at the time of surgery was $5.25 \pm 4.35$ years (range from 14 days to 11.8 years). The mean age of patients in group (A) was $5.6 \pm 3.2$ months (range: 14 days to 1 year), in group (B) was $5.8 \pm 2.3$ years (range: 1.8 to 10.8 years, and in group (C) $5.1 \pm 2.2$ years (range: 13 months to 11.8 years). All patients under age of one year had congenital cataract. Out of the 75 patients included in this study 47 patients $(63 \%)$ were males.

\section{Early Postoperative Complications:}

Early postoperative complications (defined as complications recognized in the first 3 postoperative days) were diagnosed in 34 eyes (35.1\%). Postoperative iritis occurred in 7 eyes $(7.2 \%)$ with mild inflammatory reaction. All affected eyes treated with more frequent topical prednisolone acetate 1\%. Striate keratopathy was found in 4 eyes $(4.12 \%)$ and cleared after frequent topical steroid application. Postoperative endophthalmitis: occurred in one eye in group A in the second postoperative day and treated immediately by intravitreal injection of antibiotic. Twenty-four hours later, 3 ports pars plana vitrectomy was done but unfortunately the eye passed into atrophia. Early IOL capture occurred in 2 eyes $(2.1 \%)$ with PMMA IOL. Both patients belonged to group $\mathrm{C}$. Both occurred in the second postoperative day. The capture was managed successfully by mydriasis in the 2 cases. Hyphema occurred in 4 eyes $(4.12 \%), 2$ eyes $(2.1 \%)$ in group A, $1(1 \%)$ in group B and $1(1 \%)$ in group C. In all cases, the hyphema spontaneously resolved within 3 days.

Table 1 show early postoperative complications in the study

\begin{tabular}{|c|c|c|c|c|}
\hline Complication & $\begin{array}{c}\text { Group (A) } \\
\text { No. (\%) }\end{array}$ & $\begin{array}{c}\text { Group (B) } \\
\text { No. }(\boldsymbol{\%})\end{array}$ & $\begin{array}{c}\text { Group (C) } \\
\text { No. }(\boldsymbol{\%})\end{array}$ & Total \\
\hline Hyphema & $\mathbf{2 ( 2 . 1 )}$ & $\mathbf{1 ( 1 )}$ & $\mathbf{1 ( 1 )}$ & $\mathbf{4 ( 4 . 1 2 )}$ \\
\hline Fibrinous reaction & $\mathbf{4 ( 4 . 1 2 )}$ & $\mathbf{2 ( 2 . 1 )}$ & $\mathbf{1 ( 1 )}$ & $\mathbf{7 ( 7 . 2 2 )}$ \\
\hline Striate keratopathy & $\mathbf{2 ( 2 . 1 )}$ & $\mathbf{1 ( 1 )}$ & $\mathbf{0 ( 0 )}$ & $\mathbf{3 ( 9 . 3 )}$ \\
\hline Endophthalmitis & $\mathbf{1 ( 1 )}$ & $\mathbf{0 ( 0 )}$ & $\mathbf{0 ( 0 )}$ & $\mathbf{1 ( 1 )}$ \\
\hline Capture IOL & $\mathbf{0 ( 0 )}$ & $\mathbf{2 ( 2 . 1 )}$ & $\mathbf{0 ( 0 )}$ & $\mathbf{2 ( 2 . 1 )}$ \\
\hline Total & $\mathbf{1 1}$ & $\mathbf{6}$ & $\mathbf{2}$ & $\mathbf{1 7}$ \\
\hline
\end{tabular}

Late postoperative complications:

Postoperative retinal detachment (RD) occurred in one eye (1\%) in group B and managed by pars plana vitrectomy and silicon oil injection which was removed 6 months later. Ultrasound was done and confirmed attached retina during the follow up period. The best corrected visual acuity at the end of the study was 5/60. Secondary glaucoma occurred in 8 eyes (8.2\%); 3 aphakic (3.1\%) and 5 pseudophakic (5.1\%). Intraocular pressure (IOP) was controlled medically during the study period; medically (dorzolamide and timolol combination) in 6 eyes $(6.1 \%)$ and by trabeculectomy in the remaining 2 eyes $(2.1 \%)$.

Posterior capsule opacification (PCO) occurred in 30 eyes $(31 \%)$ during the first 3 months of follow up. Seven eyes (7.2\%) belonged to group A. In group B, 5 eyes $(5.1 \%)$ had PCO; all with PMMA IOL; no PCO occurred in the 16 eyes that were implanted acrylic IOL and the optic of the IOL was captured into the posterior capsulorrehxis. For group C, PCO was recognized in 18 eyes (18.5\%); 10 eyes with PMMA IOL and 8 eyes with acrylic IOL. Sixteen eyes with PCO (16.5\%) were managed by YAG posterior capsulotomy during the first 6 months postoperatively. These included 2 eyes $(2.1 \%)$ in group B, and $14(14.4 \%)$ in group C. The remaining 
14 eyes required surgical posterior capsulotomy; 7 (7.2\%) in group A, 3 (3.1\%) in group B and $4(4.1 \%)$ in group C.

Late IOL capture occurred in 9 eyes $(9.3 \%)$. These included 5 eyes $(5.2 \%)$ in group B; among them 4 eyes $(4.1 \%)$ had been implanted PMMA IOL and were successfully managed by mydriasis in $2(2 \%)$ and by surgical repositioning in the other $2(2 \%)$. The fifth eye (1\%) had been implanted foldable IOL and was managed successfully by mydriasis. In group C, late IOL capture occurred in 4 eyes (4.1\%), among them 3 eyes $(3.1 \%)$ had been implanted PMMA IOL and were successfully managed by mydriasis in one eye $(1 \%)$, and surgically in another eye $(1 \%)$ while no intervention was done in the third eye due to family refusal. The fourth eye $(1 \%)$ had been implanted foldable IOL and was successfully managed by mydriasis.

Table 2 show late postoperative complication

\begin{tabular}{|c|c|c|c|c|}
\hline Complication & $\begin{array}{c}\text { Group (A) } \\
\text { No. }(\%)\end{array}$ & $\begin{array}{c}\text { Group (B) } \\
\text { No. }(\%)\end{array}$ & $\begin{array}{c}\text { Group (C) } \\
\text { No. }(\%)\end{array}$ & $\begin{array}{c}\text { Total } \\
\text { No. }(\%)\end{array}$ \\
\hline $\begin{array}{c}\text { Retinal } \\
\text { detachment }\end{array}$ & $\mathbf{0}(\mathbf{0})$ & $1(1)$ & $\mathbf{0}(\mathbf{0})$ & $1(1)$ \\
\hline $\begin{array}{c}\text { Secondary } \\
\text { glaucoma }\end{array}$ & $3(3.1)$ & $3(3.1)$ & $2(2)$ & $8(8.2)$ \\
\hline PCO & $7(7.2)$ & $5(5.2)$ & $18(18.5)$ & $30(30.9)$ \\
\hline Capture IOL & $\mathbf{0}(\mathbf{0})$ & $4(4.1)$ & $5(5.2)$ & $9(9.3)$ \\
\hline Total & $10(10.3)$ & 13 & 25 & 48 \\
\hline
\end{tabular}

\section{Discussion}

Management of childhood cataract remains a challenge and is complicated by the fact that it requires special surgical skills, tends to have more inflammation, and over a period of time has a risk of amblyopia along with change in refractive state of the eye. But in our series, all the children had a significant improvement over their pre-operative visual acuity, included those who were operated late ${ }^{(5)}$.

Regarding early postoperative complications, fibrinous reaction and hyphema was most common representing $11 \%$ for each. These results cope with that of Stager et al. ${ }^{(5)}$ who reported that early postoperative fibrinous reaction occurred in $13.3 \%$. They also reported that one eye lost vision to central steady and maintained fixation CSMF due to early postoperative endophthalmitis like our results that one case pass to atrophia due to early posterative endophthalmitis, also had similar results to the current study regarding postoperative retinal detachment that occurred in one case ${ }^{(5)}$.

In the current study, the most common postoperative complication was PCO in $30 \%$ of cases similar to Stager et al. ${ }^{(5)}$ who reported that PCO occurred in $27.4 \%$ of cases. Postoperative glaucoma develops in 8 eyes $(8.2 \%) 6$ of them controlled mediacally and the remaining 2 eyes needed surgical management in the form of trabeculectomy.

Vishwanath et al. ${ }^{(6)}$ reported that bilateral lensectomy during the first month of life is associated with a higher risk of subsequent glaucoma than surgery performed later. They suggested that it may be prudent, in bilateral cases, to consider delaying surgery until the infant is 4 weeks old. Trivedi et al. ${ }^{(7)}$ concluded that patients undergoing cataract surgery with or without IOL implantation at an early 
age are at high risk for development of glaucoma. Swamy et al. ${ }^{(8)}$ reported secondary glaucoma as an important sequela following surgery for congenital cataracts. They concluded that these patients should have lifelong surveillance, as glaucoma can occur years after surgery.

Michaelides et al. (9) also reported that early surgery in patients with bilateral cataracts is associated with a marked increase in risk of AG. They suggested that an intact posterior capsule may be associated with a lower rate of $A G$.

Khan. (10) noted that the lowest relative risk for later $\mathrm{AG}$ in their cohort was for surgery performed at 3-4 months of age. Kirwan et al. ${ }^{(11)}$ noted that surgery for congenital cataract at an early age increases the risk of glaucoma development, regardless of whether the eye is aphakic or pseudophakic. Ross et al. ${ }^{(\mathbf{1 2})}$ in a 20year retrospective study, noted that factors other than age at surgery are important risk factors for glaucoma in these eyes.

\section{Conclusion}

Complications after cataract surgery in children are often associated with a robust inflammatory reaction or secondary opacity and, in infants, glaucoma. Late complications can occur decades later, so that long-term follow-up is required. Though surgery carries significant risks, the consequences of no surgery and irreversible deprivation amblyopia in very young children should be considered.

\section{References}

1. Vasavada AR, Praveen MR, Nath

$\mathrm{V}$, Dave K. Diagnosis and management of congenital cataract with preexisting posterior capsule defect. Journal of cataract and refractive surgery. 2004;30(2):403-8.
2. Chen MS, Ye YJ, Wang Y, Liu Y, Wu L, Luo WX. [Posterior continuous curvilinear capsulorhexis with optic capture of the posterior chamber intraocular lens in pediatric cataract]. [Zhonghua yan ke za zhi] Chinese journal of ophthalmology. 2006;42(5):400-2.

3. Bar-Sela SM, Spierer A. Astigmatism outcomes of scleral tunnel and clear corneal incisions for congenital cataract surgery. Eye. 2006;20(9):1044-8.

4. Weisberg OL, Sprunger DT, Plager DA, Neely DE, Sondhi N. Strabismus in pediatric pseudophakia. Ophthalmology. 2005;112(9):1625-8.

5. Stager DR, Jr., Wang X, Weakley DR, Jr., Felius J. The effectiveness of Nd:YAG laser capsulotomy for the treatment of posterior capsule opacification in children with acrylic intraocular lenses. Journal of AAPOS : the official publication of the American Association for Pediatric Ophthalmology and Strabismus. 2006;10(2):159-63.

6. Vishwanath M, Cheong-Leen R, Taylor D, Russell-Eggitt I, Rahi J. Is early surgery for congenital cataract a risk factor for glaucoma? Br J Ophthalmol. 2004;88(7):905-10.

7. Trivedi RH, Wilson ME, Jr., Golub RL. Incidence and risk factors for glaucoma after pediatric cataract surgery with and without intraocular lens implantation. Journal of AAPOS : the official publication of the American Association for Pediatric Ophthalmology and Strabismus. 2006;10(2):117-23.

8. Swamy BN, Billson F, Martin F, Donaldson C, Hing S, Jamieson R, et al. Secondary glaucoma after paediatric cataract surgery. $\mathrm{Br} \quad \mathrm{J}$ Ophthalmol. 2007;91(12):1627-30.

9. Michaelides M, Bunce C, Adams GG. Glaucoma following congenital cataract surgery--the role of early 
SOHAG MEDICAL JOURNAL Postoperative complications (early and late) after pediatric

surgery and posterior capsulotomy. BMC Ophthalmol. 2007; 7:13.

10. Khan AO. Emmetropization after lensectomy and anterior vitrectomy for persistent hyperplastic primary vitreous cataract. Eye Contact Lens. 2007;33(4):199-200.

11. Kirwan C, Lanigan B, O'Keefe M. Glaucoma in aphakic and pseudophakic eyes following surgery for congenital cataract in the first year of life. Acta ophthalmologica. 2010;88(1):53-9.

12. Ross A, Ross AH, Mohamed Q. Review and update of central serous chorioretinopathy. Current opinion in ophthalmology. 2011;22(3):16673. 